\title{
Innovation and Validation of Neuropsychopharmacological Testing Methods for the Assessment of Memory, Attention and Cognition in Human Participants
}

\author{
Md. Sahab Uddin*, Md. Asaduzzaman \\ Department of Pharmacy, Southeast University, Dhaka, Bangladesh \\ Email: *msu-neuropharma@hotmail.com, msu_neuropharma@hotmail.com
}

Received 2 March 2016; accepted 20 July 2016; published 22 July 2016

Copyright (C) 2016 by authors and Scientific Research Publishing Inc.

This work is licensed under the Creative Commons Attribution International License (CC BY). http://creativecommons.org/licenses/by/4.0/

c. (i) Open Access

\section{Abstract}

To meet the challenges of time searching for the new method for neuropsychopharmacology is still an ongoing process. In this experiment, we proposed and validated three neuropsychopharmacological testing methods, which are Matching Capacity (MC), Dissimilarity Identification (DI) and Sense Making (SM) tests for the estimation of memory, attention and cognition respectively in healthy aged human participants. The purpose of this experiment was to generate more effective testing methods for the determination of memory, attention and cognition in human. To endorse proposed methods a randomized double blind placebo controlled clinical trial was conducted among healthy aged human participants between the period of December 2015 and February 2016. The experiment was conducted at Gazipur Sadar, Gazipur-1703, Dhaka, Bangladesh and the experiment center was the Department of Pharmacy, Southeast University, Dhaka-1213, Bangladesh. In this experiment, 60 healthy male participants between the age of 42 to 50 years were selected and divided into 4 groups with 15 participants in each by simple randomization process. Ginkgo biloba standardized extract's capsule (Giloba ${ }^{\circledR}$ ) and Bacopa monnieri standardized extract's syrup (Monera ${ }^{\circledR}$ ) were used as standard. Psyllium seed husk (i.e., seed of the Plantago ovata) was incorporated in the hard gelatin capsule shell (size 0 ) to prepare capsule and used as a placebo control of the capsule (PCC). Excipients of oral liquid preparation of vitamin were used to prepare syrup and used as a placebo control of the syrup (PCS). The treatment procedure for group 1 was Ginkgo biloba standardized extract's capsule $(120 \mathrm{mg} /$ capsule $), 3$ times daily for 21 days; group 2 was treated with Bacopa monnieri standardized extract's syrup $(1.21 \mathrm{~g} / 5 \mathrm{ml}), 2$ teas-

Corresponding author.

How to cite this paper: Uddin, Md.S. and Asaduzzaman, Md. (2016) Innovation and Validation of Neuropsychopharmacological Testing Methods for the Assessment of Memory, Attention and Cognition in Human Participants. Neuroscience and Medicine, 7, 83-98. http://dx.doi.org/10.4236/nm.2016.73010 
poonfuls, 3 times daily for 21 days. Psyllium seed husk's capsule and excipients of oral liquid vitamin's syrup were used as placebo control (PC) for group 3 and group 4 and administered in the similar manner of group 1 and group 2, respectively. Administration of Ginkgo biloba (GB) and Bacopa monnieri (BM) standardized extracts for successive days significantly increased the memory, attention and cognition of the participants with respect to PC groups. In the MC test, treatment with GB extract markedly $(P<0.05)$ increased the memory of the participants on $21^{\text {st }}$ day with respect to PCC group. On the other hand administration of BM extract remarkably $(P<0.01)$ increased the memory of the participants on $14^{\text {th }}$ day in comparison with PCS group. For DI test, administration of GB extract noticeably $(P<0.01, P<0.05)$ increased the attention of the participants on $14^{\text {th }}$ and $21^{\text {st }}$ day as compared to that of PCC group. Conversely BM extract significantly $(P$ $<0.05, P<0.01)$ increased the attention of the participants on $7^{\text {th }}, 14^{\text {th }}$ and $21^{\text {st }}$ day with regard to PCS group. In the SM test, administration of GB extract noticeably $(P<0.05)$ increased the cognition of the participants on $7^{\text {th }}$ and $21^{\text {st }}$ day in comparison with PCC group. BM extract administration considerably $(P<0.01)$ increased cognition of the participants on $14^{\text {th }}$ and $21^{\text {st }}$ day related to the PCS groups. The experiment demonstrates that MC, DI and SM tests clearly exposed the memory, attention and cognition enhancing activity of the GB and BM standardized extracts in healthful aged human participants respectively. Therefore, the proposed method can be used for the fortitude of memory, attention and cognition in human and will generate a landmark in the sphere of neuroscience.

\section{Keywords}

Neuropsychopharmacology, Matching Capacity Test, Dissimilarity Identification Test, Sense Making Test, Memory, Attention, Cognition, Clinical Trial

\section{Introduction}

The extraordinary feature of the human being is owing to the presence of the brain [1]. This three-pound brain is the center of all functions that we do [2]. There is no relationship between the size of the brain and the intelligence within a species. In fact the brain size of Albert Einsteins was on average, smaller than aged matched controls [3]. After all, brain size does correlate with differences in intelligence between species, although some adjustment with body surface is vital [4]. Most of the size of the human brain arises from the cerebral cortex, especially from the frontal lobes, which are linked with cognitive functions [5].

Memory is a set of programmed neural connections in the brain with the intention of retention and retrieves of information and past experiences. Physiologically, memories are stored in the brain as a result of previous neural activity by changing the basic sensitivity of synaptic transmission between neurons [6]. Attention is the behavioral and cognitive process of carefully concentrating on specific information by ignoring other perceivable information. It has also been referred to the act of close or careful observing or listening [7]. Many studies suggest that during attention the brain is incapable of multitasking. Cognition is an intellectual process related to perception, attention, memory, motor skills, language, visual and spatial processing, executive functions etc. [8]. Increasing age of the world's population is responsible for suffering from neurological and psychological disorders. With age, some cognitive functions have a tendency to decline, particularly executive functions and those cognitive skills that are not used regularly. In addition the aging is linked with a greatly increased incidence of a number of neurodegenerative disorders such as Alzheimer's disease (AD), Parkinson's disease (PD), amyotrophic lateral sclerosis (ALS) etc. [9].

Worldwide neurological disorders are the leading causes of disease burden [10]. All over the world about 100 millions of people are affected by neurological disorders [11]. In 2005 neurological disorders contributed to 92 million disability-adjusted life-years (DALYs) and were expected to 103 million in 2030 [12]. In order to study cognitive deficits in patients with neurological and or psychological or neuropsychological disorders, various behavioral tests are effective [13]. Behavioral tests are also effective for the determination of the nootropic activity of medicinal plants/drugs [14]. However, most of the tests are based on traditional psychometric principle 
and focus on animal model. Therefore, the objective of this experiment was to show and prove three neuropsychopharmacological testing methods, which are Matching Capacity (MC), Dissimilarity Identification (DI) and Sense Making (SM) tests for the determination of memory, attention and cognition respectively in healthy aged human participants.

\section{Objectives}

The specific objectives of the MC, DI and SM tests are presented below:

1 . To resolve memory problems in neurological and or psychological or neuropsychological patients MC test is effective.

2. To determine the attention deficits in neurological and or psychological or neuropsychological patients DI test is operative.

3. To measure the cognitive impairment in neurological and or psychological or neuropsychological patients SM test is capable.

4. To ensure the memory enhancing activity of unknown medicinal plants/drugs in human MC test is competent.

5. To confirm the effect of unknown medicinal plants/drugs in attention of human DI test is feasible.

6. To expose the cognition enhancing activity of unknown medicinal plants/drugs in human SM test is active.

7. To endorse the nootropic activity of the established drugs in human MC, DI and SM tests is efficient.

8. To validate the memory, attention and cognition of human MC, DI and SM tests is useful respectively.

\section{Materials and Methods}

\subsection{Study Design}

This was a randomized double blind placebo controlled clinical trial. A flow chart of the study protocol is presented in Figure 1. The participants of this experiment were randomized by simple randomization process. This study was conducted at Gazipur Sadar, Gazipur-1703, Dhaka, Bangladesh between the period of December 2015 and February 2016. The experiment center was the Department of Pharmacy, Southeast University, Dhaka-1213, Bangladesh. The protocol of the experiment was approved by the ethics committee of the Department of Pharmacy, Southeast University, Dhaka-1213, Bangladesh.

\subsection{Study Population}

Healthy 60 male participants between the age of 42 to 50 years were randomly recruited and finally all participants took part in the end of the experiment. Demographic information of the participants is given in Table 1. Prior to the experiment written informed consent was obtained from each participant. To evaluate the health status of the participants before the selection of the participants, they were interviewed with a complete set of health related questions.

\section{Inclusion and Exclusion Criteria}

The participants were included in the experiment if they met the following conditions:

1. No previous history of neurological disorders.

2. No previous history of psychological disorders.

3. No history of drug addiction.

4. No history of severe illness within the previous 3 months.

5. Free from cardiovascular, renal, hepatic, eye disease and diabetes.

6. Participants who will miss the doses for more than one day were planned to exclude from the experiment.

In this experiment factors like lifestyle, work profile, work stress, food habits, medication history, socioeconomic condition, personal issues and intelligence quotient of the participants were considered before selection of the participants. The selection of participants also considered the educational background and body surface area that were not to differ too much within the selected participants.

It is not possible and unethical to create cognitive impairment in human to validate mentioned methods. It is also unlikely to choose participants with cognitive impairment (i.e., $\mathrm{AD}$ ) who are able to participate in this experiment. Aging is strongly connected to oxidative stress. Several studies have suggested that aged people are 


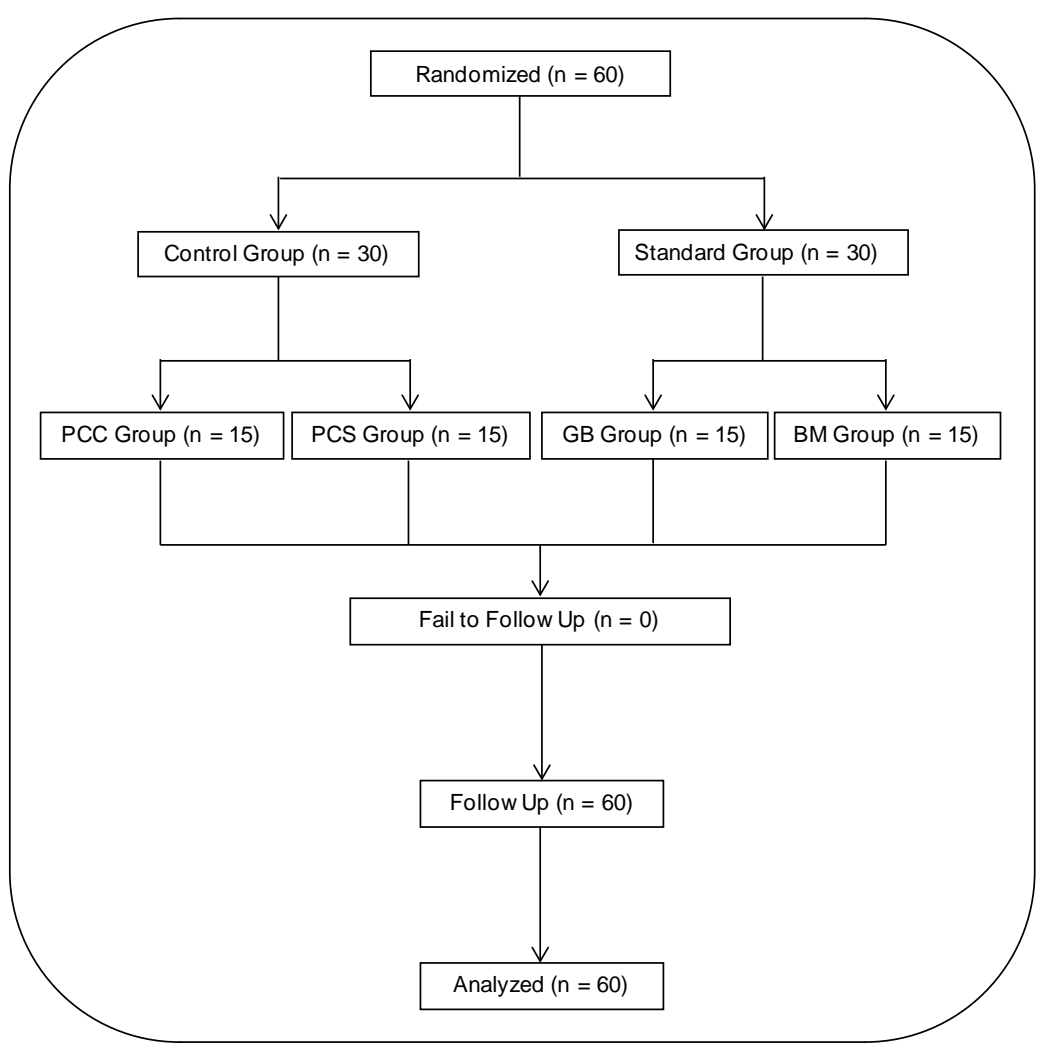

Figure 1. Outline showing the movement of the participants through the clinical trial.

Table 1. Demographic information of the participants.

\begin{tabular}{lcc}
\hline \multicolumn{1}{c}{ Variable } & $\mathrm{n}$ & $\%$ \\
\hline $\begin{array}{l}\text { Sex } \\
\text { Male }\end{array}$ & 60 & 100 \\
Age & & \\
$\quad 42-45$ & 36 & 60.00 \\
$46-48$ & 15 & 25.00 \\
$49-50$ & 9 & 15.00 \\
Education Level & & \\
$<$ SSC & 11 & 18.33 \\
$\quad$ SSC & 26 & 43.33 \\
HSC & 13 & 21.67 \\
$\quad$ Degree (pass) & 10 & 16.67 \\
Occupation & & \\
Office worker & 24 & 40.00 \\
Business & 16 & 26.67 \\
Teacher & 12 & 20.00 \\
Others & 8 & 13.33 \\
Residential Status & & \\
Residential & 41 & 68.33 \\
Nonresidential & 19 & 31.67 \\
\hline
\end{tabular}

Where, SSC = Secondary school certificate, HSC $=$ Higher secondary certificate, Degree $($ Pass $)=$ This is considered to be a pass but without the honours. 
more prone to oxidative stress that lead to neurological disorders, neurodegenerative disease, dementia, cognitive impairment than younger one [15]-[17]. Therefore, in this experiment healthy aged participants were chosen for validation of the proposed methods.

\subsection{Standard Drugs}

Ginkgo biloba (GB) and Bacopa monnieri (BM) are well-known recognized natural nootropic herbal drug [18]. In this experiment to examine the efficacy of the proposed testing methods GB and BM standardized extracts were used as standard drug. GB leaf extracts contain numerous active constituents including flavonoids, terpenoids and organic acids. Most of the standardized GB leaf extract contains $24 \%$ to $25 \%$ flavone glycosides and $6 \%$ terpene lactones. The other compounds presence in this extract is proanthocyanidins (7\%), carboxylic acids (13\%), catechins (2\%), non-flavonol glycosides (20\%) etc. [19]. The active constituents presence in BM is alkaloids (brahmine and herpestine), flavonoids (glucuronyl-7-apigenin, glucuronyl-7-luteolin, luteolin-7-glucoside and luteolin), saponins (bacogenins, bacosides and bacopasides) and bacopasaponins. Additionally, this plant contains hersaponin, monnierin and triterpines ( $\beta$-Sitosterol, stigmastanol, stigmasterol, betulic acid and bacosine) [20]. A recent study suggested that BM standardized extract that contain not less than $55 \%$ bacosides has positive nootropic effects [21]. Research shows that GB and BM are effective to improve memory, attention span and cognitive function [22] [23].

Widely used and commercially available preparations of GB and BM standardized extracts in Bangladesh are Giloba $^{\circledR}$ and Monera ${ }^{\circledR}$ manufactured by SQUARE Herbal \& Nutraceuticals Ltd. The dosage form of Giloba ${ }^{\circledR}$ is capsule and Monera ${ }^{\circledR}$ is available as syrup. For this study Giloba ${ }^{\circledR}$ and Monera ${ }^{\circledR}$ were purchased from retail pharmacies of Dhaka city in Bangladesh.

\subsection{Treatment}

In this experiment to validate the efficacy of the proposed testing methods two standard herbal nootropic drugs were administered to participants for 21 days, these were Ginkgo biloba standardized extract (Giloba ${ }^{\circledR}$ ) and $\mathrm{Ba}$ copa monnieri standardized extract $\left(\right.$ Monera $\left.^{\circledR}\right)$. Giloba ${ }^{\circledR}$ is available as capsule that contains $120 \mathrm{mg}$ Ginkgo biloba standardized extract per capsule. Conversely Monera ${ }^{\circledR}$ is available as syrup that contain $1.21 \mathrm{~g}$ Bacopa monnieri standardized extract per $5 \mathrm{ml}$. Psyllium seed husk (i.e., seed of the Plantago ovata) was used as placebo control of capsule (PCC) and it was incorporated in the empty hard gelatin capsule shell (size 0) to prepare the capsule. Excipients that are commonly used to prepare oral liquid preparation of vitamin (i.e., sweetening agents, coloring agents, flavoring agents, preservatives, stabilizers etc.) were used as placebo control of syrup (PCS) to prepare the syrup. The capsules and syrup were manufactured in the ISO and GMP certified Modern Pharmaceuticals Ltd, Dhaka, Bangladesh. Psyllium seed husk was used as placebo control since it has no nootropic activity as well as harmless. On the other hand excipients of oral liquid preparation of vitamin are also devoid of any toxicity and convenient to administer. The duration of this experiment and doses of the these standard drugs were adjusted based on literature searches and consultation with the physician [24]-[26]. In this experiment herbal drugs were selected as standard not any allopathic drug due to the risks of excessive cholinergic activity in the participants since they were healthy aged. The clinical trial was conducted for the period of 21 days.

\subsection{Experimental Design}

The participants were randomized by simple randomization process into four groups with 15 participants in each as follows:

Group 1: This group received Ginkgo biloba standardized extract (120 mg/capsule), 1 capsule 3 times daily for 21 days (GB).

Group 2: This group received Bacopa monnieri standardized extract $(1.21 \mathrm{~g} / 5 \mathrm{ml}), 2$ teaspoonfuls 3 times daily for 21 days (BM).

Group 3: This group received psyllium seed husk (hard gelatin capsule shell size 0 ) as placebo control, 1 capsule 3 times daily for 21 days (PCC).

Group 4: This group received excipients of oral liquid preparation of vitamin as placebo control, 2 teaspoonfuls 3 times daily for 21 days (PCS). 
It was recommended to administer capsule/syrup before 30 minutes of meal for better absorption and after each 8 hours interval to remove the chance of subtherapeutic concentration due to underdose and or excessive concentration due to overdose. Participants were suggested to take standard dietary during the experiment. The health status of the participants was checked with the help of the physician after each 3 day interval. In addition to this, all participants were advised to call the experiment center if they experienced any adverse effects during the experiment. The health status of the participants was monitored for 7 days after the experiment to remove any chance of post treatment complications.

In this experiment MC, DI and SM tests were conducted among participants between the period from 10:00 am to $12: 00 \mathrm{pm}$ on $7^{\text {th }}, 14^{\text {th }}$ and $21^{\text {st }}$ day.

\subsection{Memory Test}

\section{Matching Capacity Test}

This test is based on the matching capacity of the picture. In this test a complex picture is visualized to the subject for 300 seconds presented in Figure 2. After that the picture is cut into several portions by square shaped. Then the square portions are randomly mixed and delivered to the subject for matching. The time taken by subject, to match the picture is considered as matching time (MT). This MT is effective for the determination of memory of the given subject. Gradual decrease in MT indicates progress of the memory. The duration of this test is 300 seconds. The percentage of memory retention (MR) is calculated by using the formula given below:

$$
\% \text { of MR }=\text { TNCPs } \times 100 / \text { TNPs }
$$

where, TNCPs $=$ Total number of correct portions matched by the subject, TNPs $=$ Total number of portions existing in the picture. An increase in percentage of MR is considered as an index of expanded memory.

\subsection{Attention Test}

\section{Dissimilarity Identification Test}

This test is based on the finding the dissimilarities of the pictures. In this test two identical pictures are given to the subject displayed in Figure 3. The time taken by the subject to find the dissimilarities of the picture is considered as dissimilarity finding time (DFT). Gradual decrease in DFT indicates improvement of the attention. The duration of this test is 300 seconds. The percentage of improved attention (IA) is calculated by using the formula given below:

$$
\% \text { of IA }=\text { TNCDs } \times 100 / \text { TNDs }
$$

where, TNCDs $=$ Total number of correct dissimilarities identified by the subject, TNDs $=$ Total number of dissimilarities presented in the picture. An increase in percentage of IA is considered as an index of enriched attention.

\subsection{Cognitive Test}

\section{Sense Making Test}

This test is based on the making the sense of the words. In this test letters of five words are separately placed in a random manner in a printed paper and given to the subject. The time taken by the subject to make meaningful words is considered as sense making time (SMT) specified in Figure 4. Gradual decrease in SMT indicates enhancement of the cognition. The duration of this test is 300 seconds. In order to improve the accuracy of this test only those letters are selected by which other possible words is not meaningful and or impossible. However, this task is difficult. The difficulties can be removed if a clue is given about the words. The percentage of enhanced cognition (EC) is calculated by using the formula given below:

$$
\% \text { of } \mathrm{EC}=\mathrm{TNCWs} \times 100 / \mathrm{TNWs}
$$

where, TNCWs $=$ Total number of correct words recognized by the subject, TNWs $=$ Total number of words offered in the paper. An increase in percentage of EC is considered as an index of better cognition. 


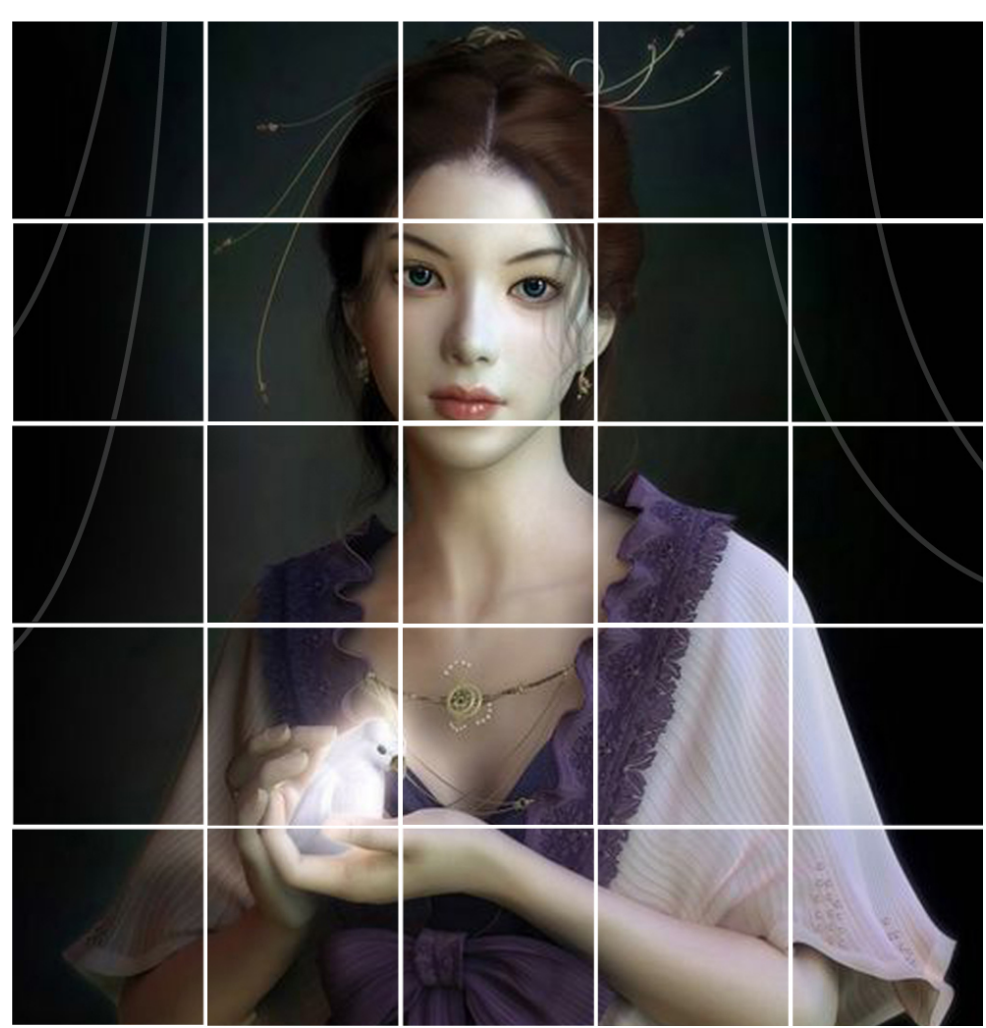

(a)

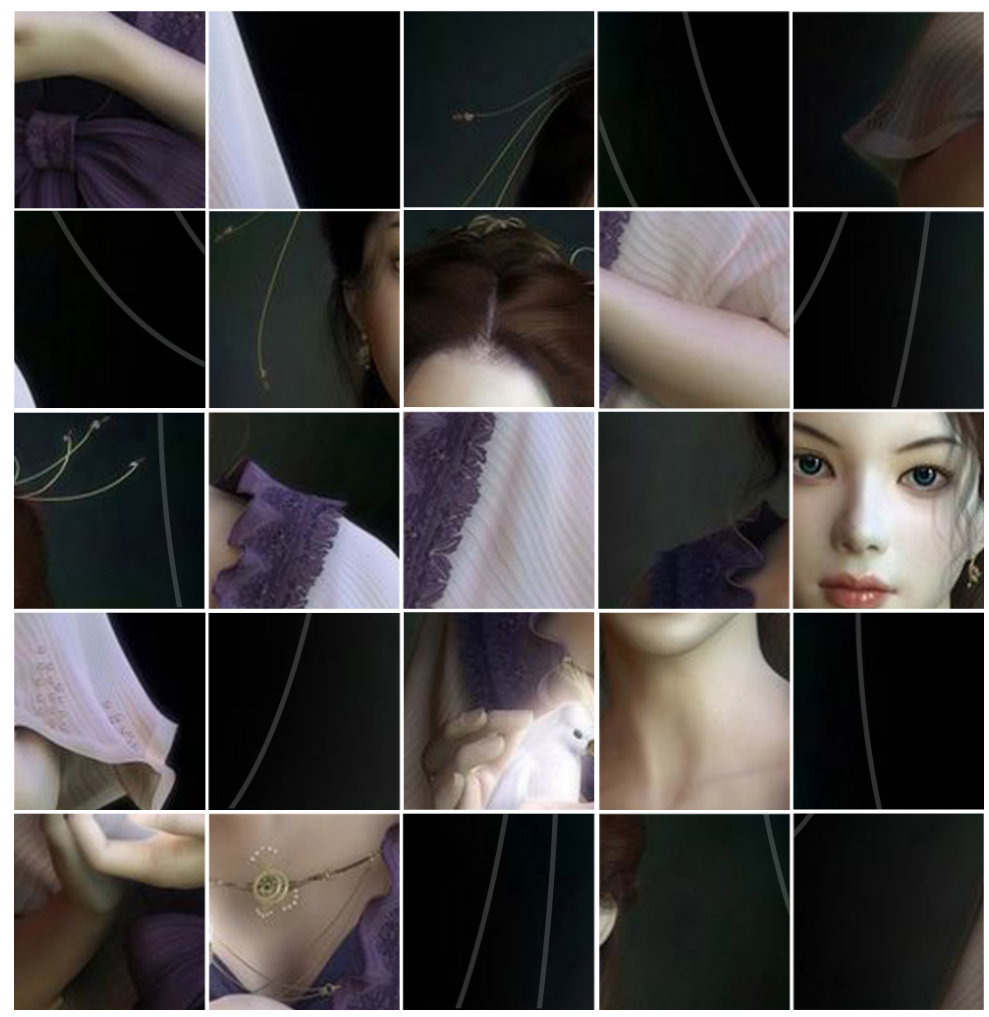

(b)

Figure 2. Matching capacity test by using picture. (a) The picture given to the subject for visual inspection; (b) Randomization of the visually inspected picture [i.e., (a)]. 


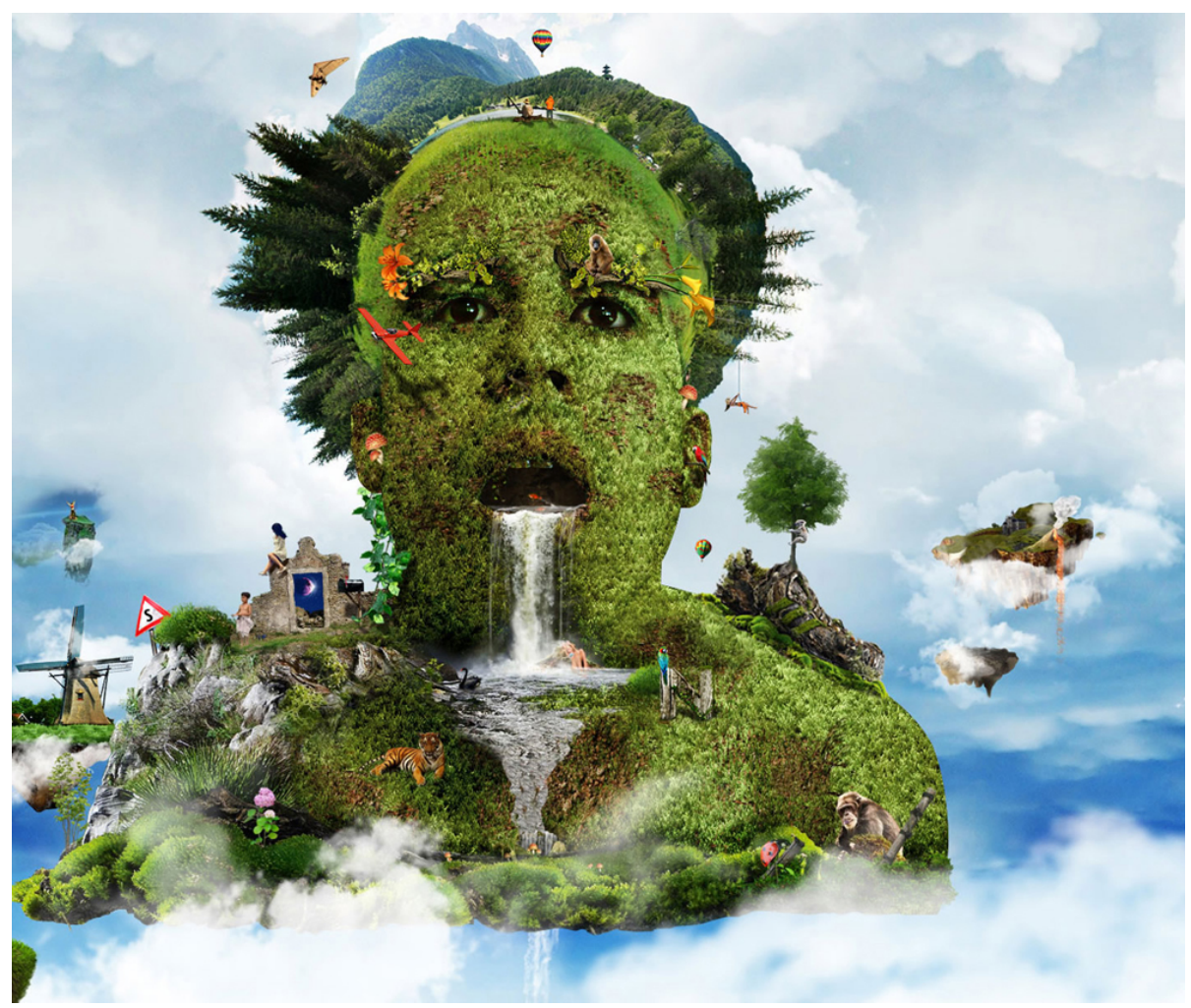

(a)

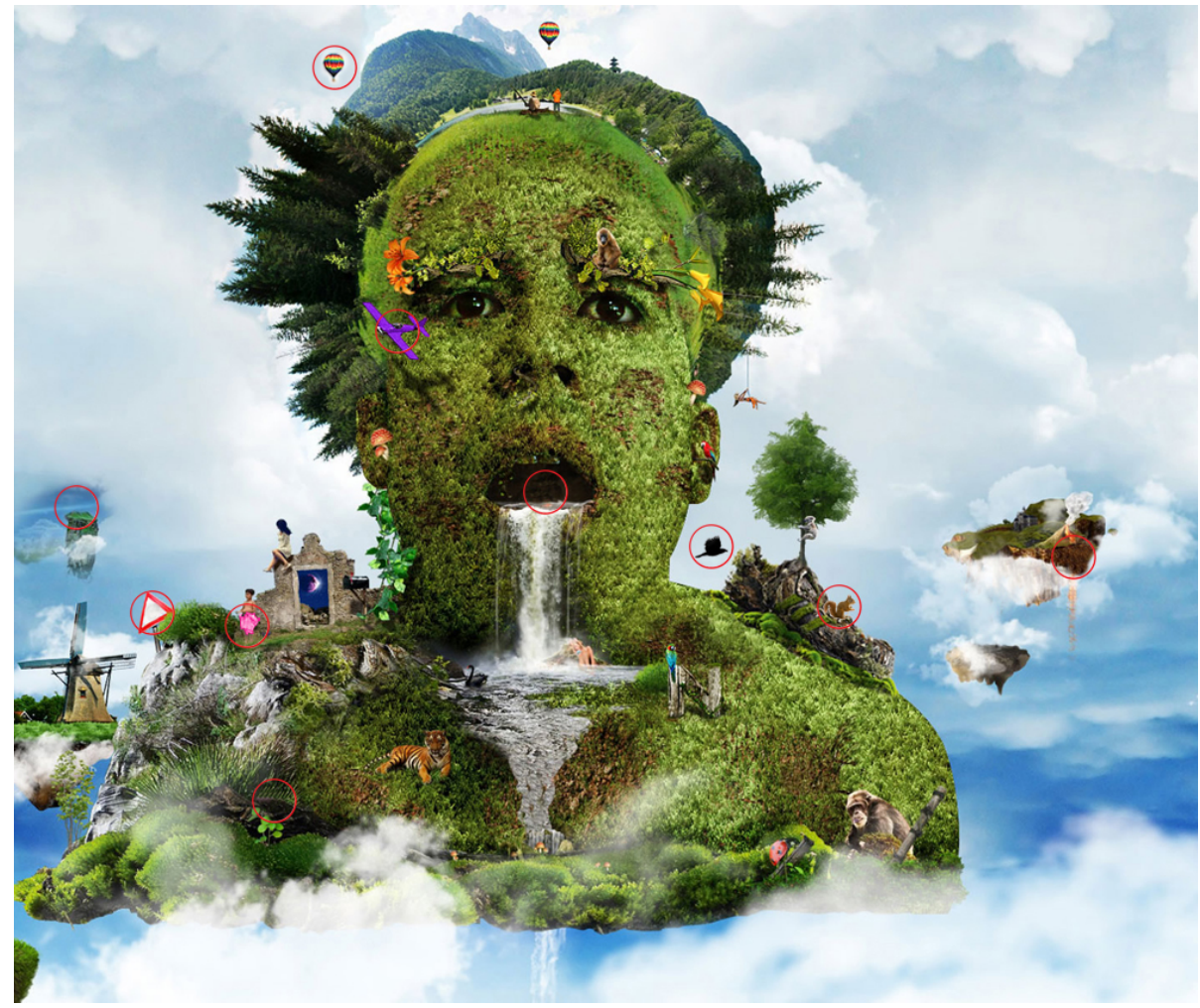

(b)

Figure 3. Dissimilarity identification test by using two identical pictures. (a) Main picture; (b) The dissimilarities of this picture with respect to main picture [i.e., (a)] are marked by the red circles. 


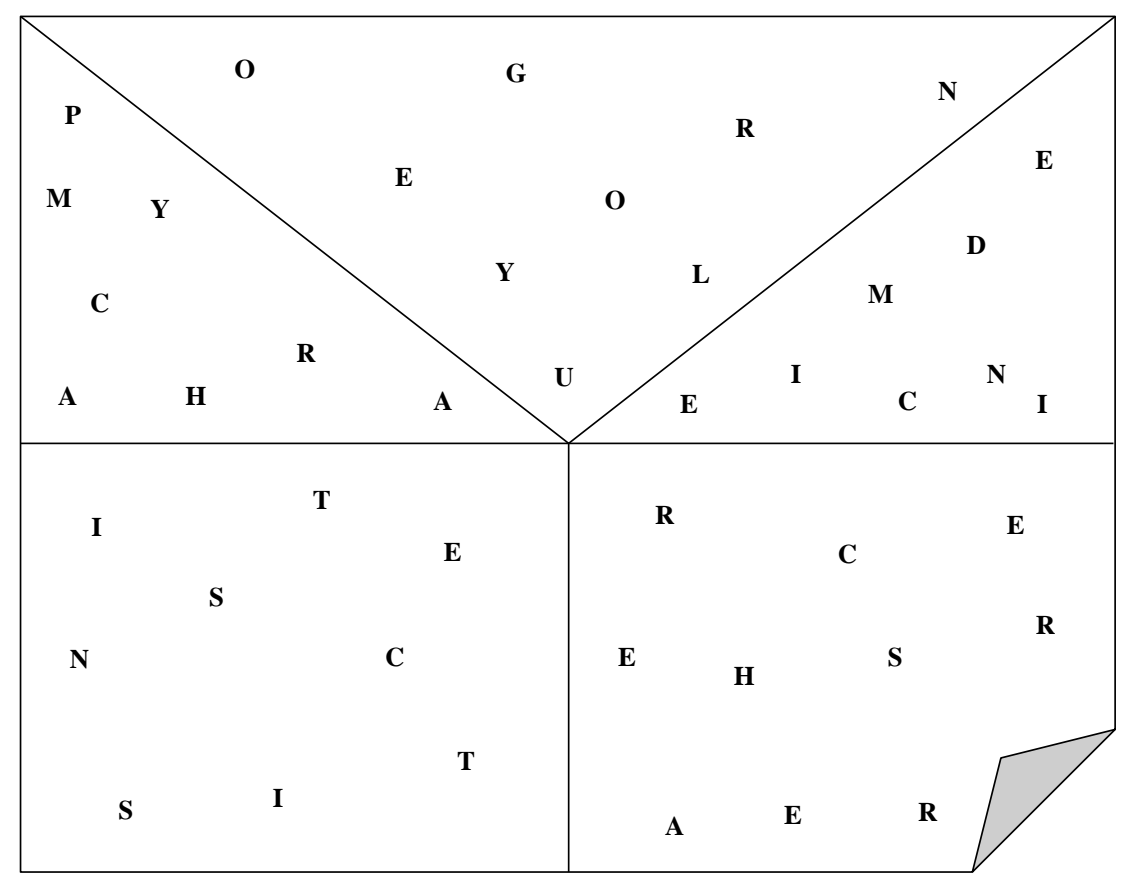

(a)

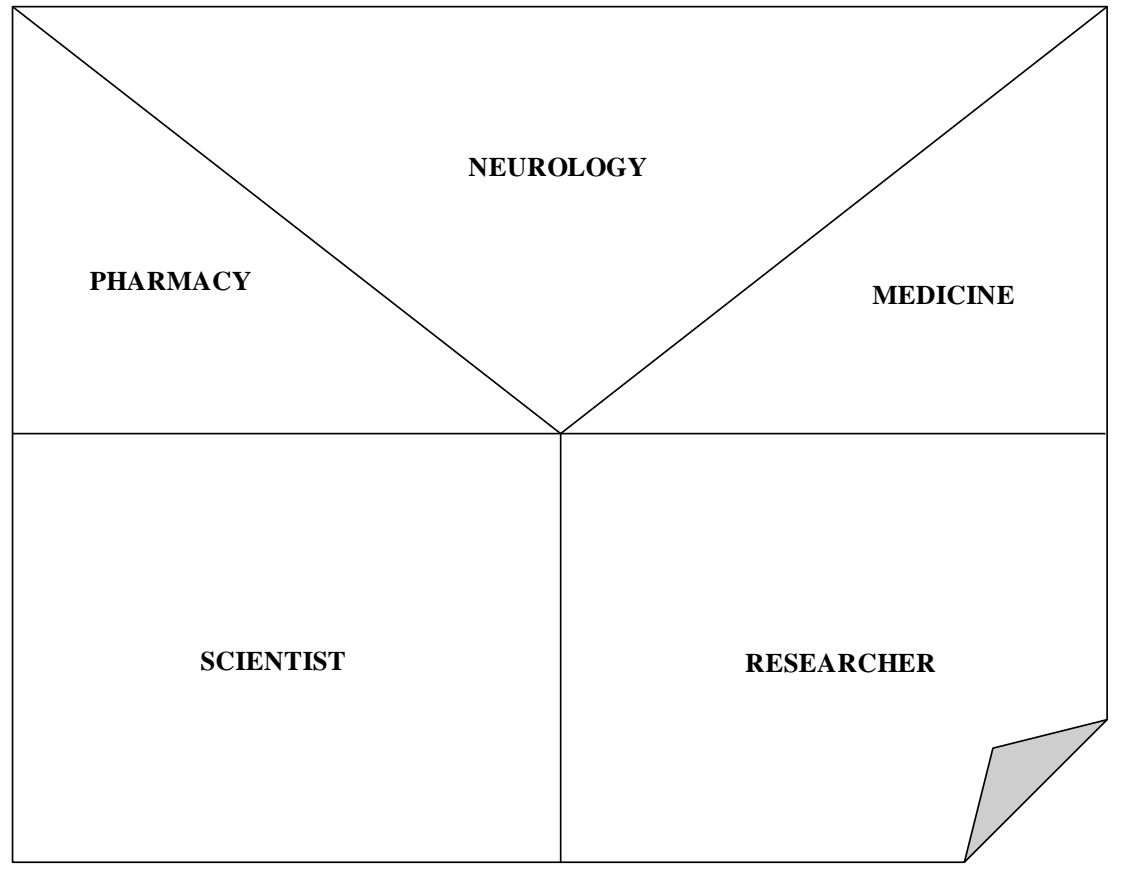

(b)

Figure 4. Sense making test by using letters to make words. (a) Randomly placed letters in a printed paper (Clue: Single word means health science); (b) Meaningful words of the randomly placed letters [i.e., (a)].

\subsection{Statistical Analysis}

All the results were expressed as mean \pm SEM and analyzed by one-way analysis of variance (ANOVA) followed by Student's t-tests for comparing between placebo control (PC) group and various treatment groups. Statistical and graphical analyses were performed using SPSS 16.0 (Chicago, IL, USA) and MS Excel 2010 (Roselle, IL, USA). The results were considered as statistically significant at $P<0.05$ with respect to PC group. 


\section{Results}

\subsection{Efficacy of the MC Test}

The results of MC test are shown in Figure 5. Administration of GB and BM standardized extracts for successive days increased the memory of the participants with respect to PC group. The GB extract significantly $(P<$ 0.05 ) increased the memory of the participants on $21^{\text {st }}$ day as compared to PCC group. Especially, administration of BM extract considerably $(P<0.01)$ increases the memory of the participants on $14^{\text {th }}$ day as compared to PCS group.

\subsection{Efficacy of the DI Test}

Figure 6 displays the results of the DI test in which administration of GB and BM standardized extract increased the attention of the participants as compared to PC group. The participants treated with the GB extract had marked $(P<0.01, P<0.05)$ increased in attention on $14^{\text {th }}$ and $21^{\text {st }}$ day with respect to PCC group. Administration of BM extract significantly $(P<0.05, P<0.01)$ increased the attention of the participants on $7^{\text {th }}, 14^{\text {th }}$ and $21^{\text {st }}$ day with respect to PCS group thus showed momentous attention enhancing activity.

\subsection{Efficacy of the SM Test}

Alteration of the cognition of the participants is displayed in Figure 7. Treatment with both GB and BM standardized extract for successive days increased the cognition of the participants to that of PC group. Administration of GB extract for successive days considerably $(P<0.05)$ increased the cognition of the participants on $7^{\text {th }}$ and $21^{\text {st }}$ day with regard to PCC group. Specifically, treatment with BM extract extensively $(P<0.01)$ increases the cognition of the participants on $14^{\text {th }}$ and $21^{\text {st }}$ day related to PCS group.

\section{Discussion}

Brain is the most convoluted part of the human body. Though scientists and philosophers over centuries tried to reveal the secret of the brain, but it remains nearly impenetrable. But at present, they have been successful in some extent to decipher the brain [27]. Owing to advancement in neurological and behavioral research, scientists

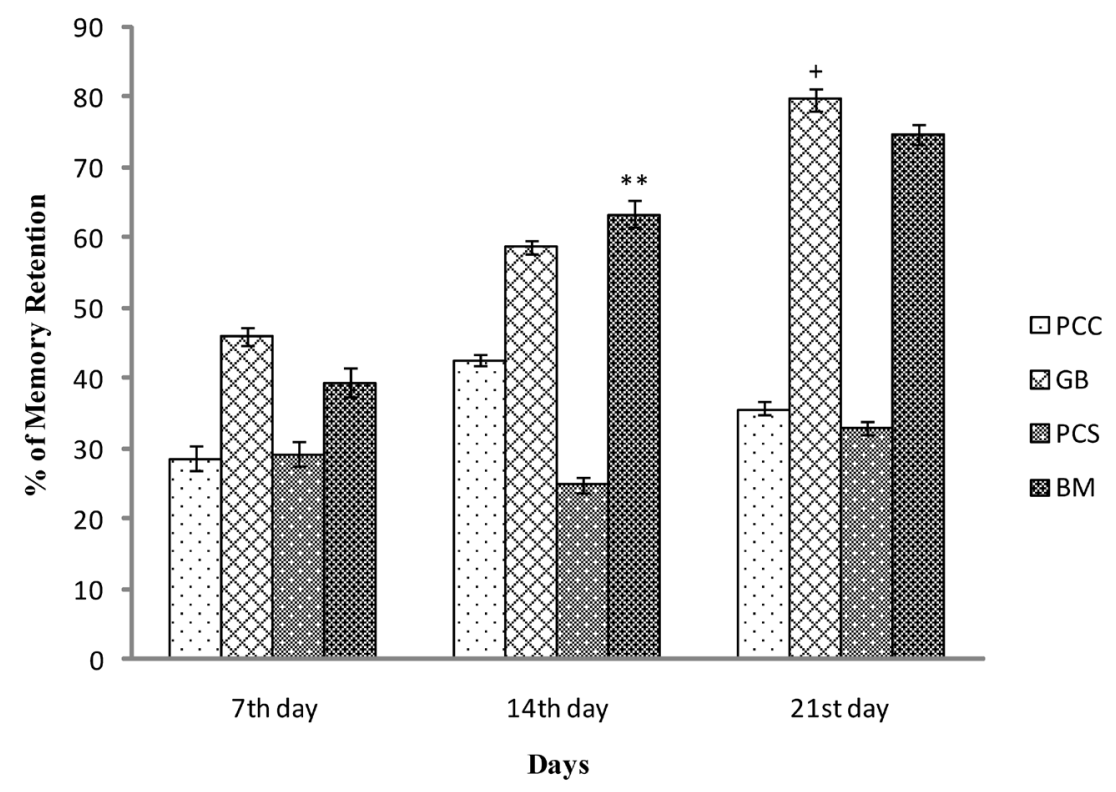

Figure 5. Effect of GB and BM on memory of human participants using MC test. Values were expressed as mean \pm SEM ( $=15$ /group). $\mathrm{GB}=$ Ginkgo biloba, $\mathrm{BM}=$ Bacopa monnieri, PCC = Placebo control of the capsule, PCS = Placebo control of the syrup. ${ }^{+} P<0.05$ significant difference from the PCC group, ${ }^{* *} P<0.01$ significant difference from the PCS group. 


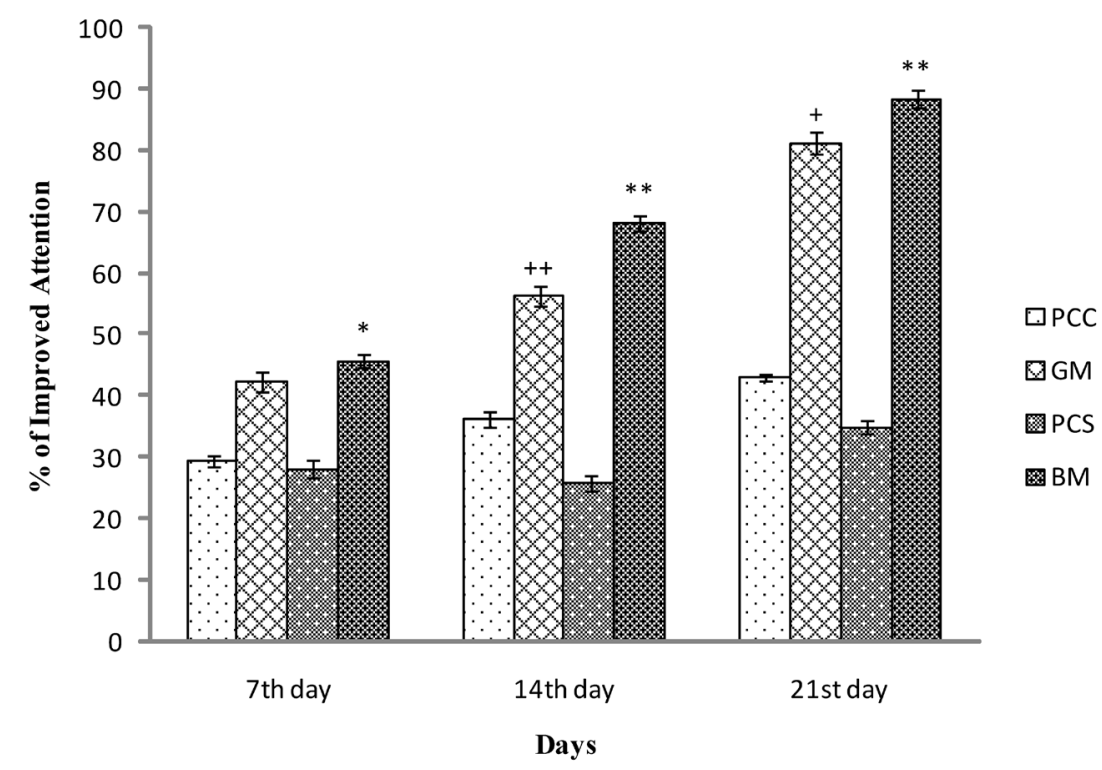

Figure 6. Effect of GB and BM on attention of human participants using DI test. Values were expressed as mean \pm SEM ( $\mathrm{n}=15$ /group). $\mathrm{GB}=$ Ginkgo biloba, $\mathrm{BM}=$ Bacopa monnieri, $\mathrm{PCC}=$ Placebo control of the capsule, PCS = Placebo control of the syrup. ${ }^{+} \mathrm{P}$ $<0.05,{ }^{++} P<0.01$ significant difference from the PCC group, ${ }^{*} P<0.05,{ }^{* *} P<0.01$ significant difference from the PCS group.

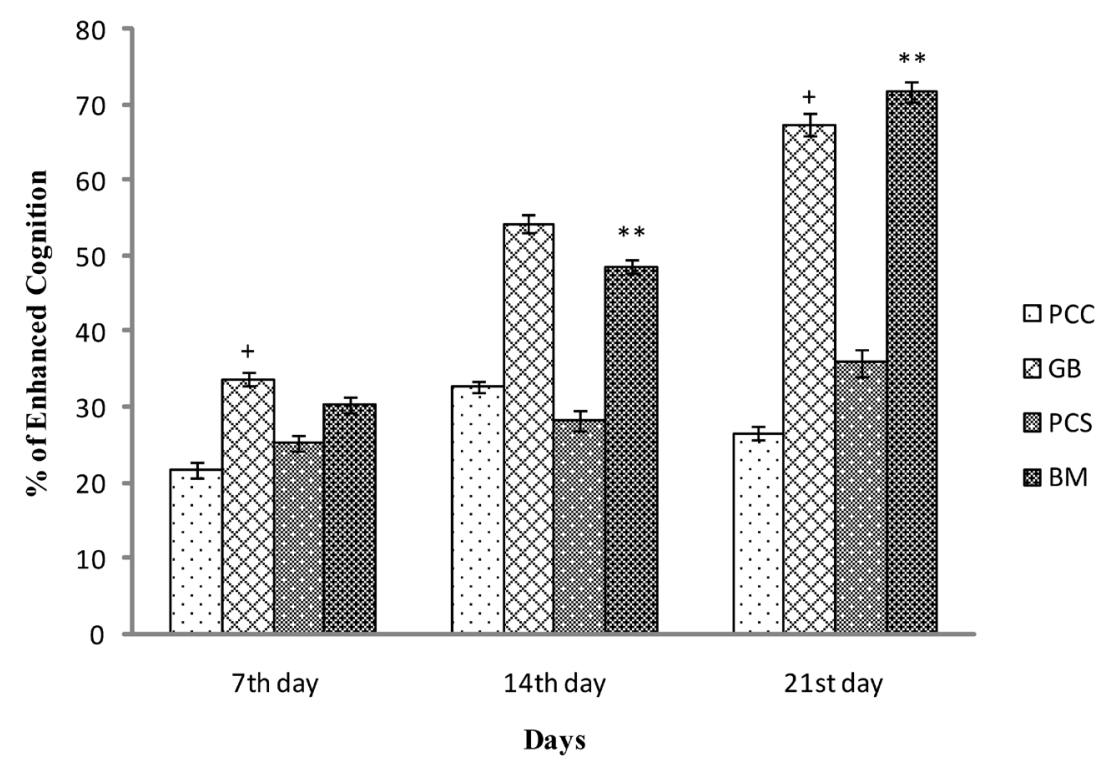

Figure 7. Effect of GB and BM on cognition of human participants using SM test. Values were expressed as mean \pm SEM ( $\mathrm{n}=15$ /group). $\mathrm{GB}=$ Ginkgo biloba, $\mathrm{BM}=$ Bacopa monnieri, PCC = Placebo control of the capsule, PCS = Placebo control of the syrup. ${ }^{+} P<0.05$ significant difference from the PCC group, ${ }^{* *} P<0.01$ significant difference from the PCS group.

have gathered more information about brain in the last decade [28]. The present experiment is represented to suggest and confirm MC, DI and SM tests for the determination of memory, attention and cognition respectively in healthy male human participants between the ages of 42 to 50 years.

All the parts of the brain work together, but each part of the brain has a unique function [29]. Most of the brain functions are regulated by the cerebrum which is the largest portion of the brain. It is divided into four 
units, for example the temporal lobe, the occipital lobe, the parietal lobe and the frontal lobe [30]. The frontal lobe is one of the four lobes in the cerebral hemisphere that is responsible for intellect, creative thought, problem solving, judgment, behavior, attention, abstract thinking, physical reactions, muscle movements, coordinated movements, smell, personality etc. [31]. The parietal lobe is positioned in the cerebral hemisphere that integrates sensory information. In addition to this, it is also responsible for monitoring visual functions, language, reading, internal stimuli, tactile sensation, sensory comprehension etc. [32]. The temporal lobe is located in the cerebral hemisphere that helps to control auditory perception, receptive components of language, visual memory, declarative (i.e., factual) memory and emotion [33]. The occipital lobe is situated in the cerebral hemisphere in the back of the head responsible to control vision [34]. In the motor control as well as some cognitive functions such as attention and language and in regulating fear and pleasure responses cerebellum plays an imperative role [35]. The limbic system contains glands that control emotional behavior and motivational drives [36].

In the daily life some memories last for only a few seconds, whereas others last for hours, days, months, or years. Actually short-term memory may last for a few seconds to a few minutes at a time, but lasting only as long as the person continues to think about the numbers or facts. Intermediate long-term memory may last for several minutes or even weeks. Actual structural changes, instead of only chemical changes, at the synapses and these enhance or suppress signal conduction is responsible for long-term memory that may last for months or years [37]. In this experiment we projected MC test and validated this test by using randomized double blind placebo controlled clinical trial for the determination of memory in aged human participants. This test reflects the memory of the image that is formed in the human brain may be caused by continual neural activity resulting from nerve signals or presynaptic facilitation or inhibition in case of short term memory or temporary chemical or physical changes or both, in either the synapse presynaptic terminals or the synapse postsynaptic membrane for intermediate long-term memory. Studies have shown that mainly temporal lobe is involved with memory [38]. MC test is more effective than Rey-Osterrieth Complex Figure test [39] which is based on drawing of the figure. Drawing is considered as gift since some are born with natural talent [40]. In addition to this drawing is a practice, anyone can learn to draw well [41]. That's why the subject with optimum memory function may not have drawn capacity [42]. However, the MC test is devoid of this type of complication, this test retrieves the memory of the given image copied within the brain.

At every moment, we are flurried with information from our surroundings. In retaining concentration, brain cells work together to arrange information according to priority from the steady stream of data and prevent the brain from being devastated [43]. DI test is recommended and endorsed by means of randomized double blind placebo controlled clinical trial for determination of attention in adult human participants. Researchers showed that inferior frontal junction (IFJ) of the prefrontal cortex controls visual processing areas that are tuned to recognize a specific category of objects [44]. In case of Letter Cancellation test one has to cross out all the target letters among randomly spread letters [45]. It's very easy test in which minimal attention is required [46]. As well as this test must be affected by educational level. As a result low educated subject represents a challenge during this assessment [47]. But DI test is free from this type of problems, to detect the dissimilarities of the given pictures comparatively more attention is essential and its not depend on education level.

Cognitive functions are brain-built skills that we need to perform any task from the simplest to the most complex. Aging cause decline of executive functions as well as rarely performed cognitive functions already stated earlier [48]. In this experiment we proposed sense making test and validated the efficacy of this test in aged human for the measurement of cognition that is based on meaningful words making ability from letters placed randomly. In the processing all of the visual information (letters) occipital lobe is very active and the frontal lobe is engaged in processing the meaning of the letters to make meaningful words [49]. SM test is superior than Stroop test [50] which is based on the names of the colors asking aptitude. Several studies exposed that women are quicker than men in naming the right color [51]. SM test has no dependency on gender. The contents of this test can be selected as per subject that's why the educational level has no influence.

The results of this experiment suggest that MC, DI and SM tests are highly active for the assessment of memory, attention and cognition enhancing power of the Ginkgo biloba and Bacopa monnieri standardized extract in healthy aged human participants respectively.

\section{Conclusion}

The human brain is a specialized organ that is ultimately responsible for all thought and movement that the body 
produces. In this experiment, we have developed MC, DI and SM tests for the determination of memory, attention and cognitive in human and validated by using a randomized double blind placebo controlled clinical trial. From the present outcome, it is undoubtedly established that MC, DI and SM tests are effective for the determination of the memory, attention and cognition enhancing power of the Ginkgo biloba and Bacopa monnieri standardized extract in healthy aged human participants respectively. So these testing methods will be effective for various neuropsychopharmacological assessments in human. The foremost feature of these aforementioned testing methods is the flexibility, which can be performed easily as per background of the subjects. Therefore, MC, DI and SM tests will create a milestone in the field of neuroscience.

\section{Limitations}

It was a pilot study. Although the participants were large enough to bring the impact it would be better if we could perform this experiment among more participants. The personality of participants may also affect the outcome of the experiment.

\section{Acknowledgements}

The authors wish to thank the participants of this experiment, our neuropsychopharmacology research team and the Department of Pharmacy, Southeast University, Dhaka-1213, Bangladesh.

\section{Consent to Participate}

The authors took consent from the participants.

\section{Consent for Publication}

The authors took consent for publication.

\section{Ethical Approval}

The study protocol was approved by the ethics committee of the Department of Pharmacy, Southeast University, Dhaka-1213, Bangladesh. This protocol was conducted in accordance with the ethical standards laid down in the 1964 Declaration of Helsinki.

\section{Author's Contributions}

This work was carried out in collaboration between the authors. Author MSU designed the methods, wrote the protocol, managed the analyses of the study and prepared the draft of the manuscript. Author MSU and MA performed the clinical trial. Author MA revised the final manuscript and enrich the scientific value of the manuscript. All the authors read and approved the final manuscript.

\section{Competing Interests}

The authors proclaim that the research was conducted in the absence of any commercial or financial relationships that could be construed as potential competing interests.

\section{References}

[1] Ghosh, D. and Parida, P. (2015) Brain of the Genius: Albert Einstein. Current Science, 108, 1787.

[2] Garg, D. and Singh, A. (2005) Soft Computing. Allied Publishers Pvt. Ltd, New Delhi.

[3] Witelson, S.F., Kigar, D.L. and Harvey, T. (1999) The Exceptional Brain of Albert Einstein. Lancet, 353, $2149-2153$. http://dx.doi.org/10.1016/S0140-6736(98)10327-6

[4] Rushton, J.P. and Ankney, C.D. (2009) Whole Brain Size and General Mental Ability: A Review. International Journal of Neuroscience, 119, 692-732. http://dx.doi.org/10.1080/00207450802325843

[5] Wikipedia (2016). https://en.wikipedia.org/wiki/Human_brain

[6] Squire, L.R. (2004) Memory Systems of the Brain: A Brief History and Current Perspective. Neurobiology of Learning 
and Memory, 82, 171. http://dx.doi.org/10.1016/j.nlm.2004.06.005

[7] Anderson, J.R. (2004) Cognitive Psychology and Its Implications. Worth Publishers, New York.

[8] Michelon, P. (2006) What Are Cognitive Abilities and Skills, and How to Boost Them? http://sharpbrains.com/blog/2006/12/18/what-are-cognitive-abilities/

[9] Marcourakis, T., Camarini, R., Kawamoto, E.M., Scorsi, L.R. and Scavone, C. (2008) Peripheral Biomarkers of Oxidative Stress in Aging and Alzheimer's Disease. Dementia \& Neuropsychologia, 2, 2.

[10] Chin, J.H. and Vora, N. (2014) The Global Burden of Neurologic Diseases. Neurology, 83, 349-351. http://dx.doi.org/10.1212/WNL.0000000000000610

[11] Burton, K.J. and Allen, S. (2003) A Review of Neurological Disorders Presenting at a Paediatric Neurology Clinic and Response to Anticonvulsant. Annals of Tropical Paediatrics, 23, 139-143. http://dx.doi.org/10.1179/027249303235002215

[12] Bower, J.H., Asmera, J., Zebenigus, M., Sandroni, P., Bower, S.M. and Zenebe, G. (2007) The Burden of Inpatient Neurologic Diseases in Two Ethiopian Hospitals. Neurology, 68, 338-342. http://dx.doi.org/10.1212/01.wnl.0000252801.61190.e8

[13] Salmon, D.P. and Bondi, M.W. (2009) Neuropsychological Assessment of Dementia. Annual Review of Psychology, 60, 257-282. http://dx.doi.org/10.1146/annurev.psych.57.102904.190024

[14] Kumar, G.P. and Khanum, F. (2012) Neuroprotective Potential of Phytochemicals. Pharmacognosy Reviews, 6, 81-90. http://dx.doi.org/10.4103/0973-7847.99898

[15] Barnes, C. and Burke, S. (2006) Neural Plasticity in the Ageing Brain. Nature Reviews Neuroscience, 7, 30-40. http://dx.doi.org/10.1038/nrn1809

[16] Feng, Y. and Xiaochuan, W. (2012) Antioxidant Therapies for Alzheimer's Disease. Oxidative Medicine and Cellular Longevity, 2012, Article ID: 472932. http://dx.doi.org/10.1155/2012/472932

[17] Vercueil, L. (2006) Epilepsy and Neurodegenerative Diseases in Adults: A Clinical Review. Epileptic Disorders, 8, S44-S54.

[18] Neale, C., Camfield, D., Reay, J., Stough, C. and Scholey, A. (2013) Cognitive Effects of Two Nutraceuticals Ginseng and Bacopa Benchmarked Against Modafinil: A Review and Comparison of Effect Sizes. British Journal of Clinical Pharmacology, 75, 728-737.

[19] Zahradníková, L., Schmidt, S., Sekretár, S. and Janáč, L. (2007) Determination of the Antioxidant Activity of Ginkgo biloba Leaves Extract. Journal of Food and Nutrition Research, 46, 15-19.

[20] Engels, G. and Brinckmann, J. (2011) Bacopa. HerbalGram, No. 91, 1-4.

[21] Pase, M.P., Kean, J., Sarris, J., Neale, C., Scholey, A.B. and Stough, C. (2012) The Cognitive-Enhancing Effects of Bacopa monnieri: A Systematic Review of Randomized, Controlled Human Clinical Trials. The Journal of Alternative and Complementary Medicine, 18, 647-652. http://dx.doi.org/10.1089/acm.2011.0367

[22] Kennedy, D.O. and Wightman, E.L. (2011) Herbal Extracts and Phytochemicals: Plant Secondary Metabolites and the Enhancement of Human Brain Function. Advances in Nutrition, 2, 32-50. http://dx.doi.org/10.3945/an.110.000117

[23] Kean, J.D., Kaufman, J., Lomas, J., Goh, A., White, D., David Simpson, D., et al. (2015) A Randomized Controlled Trial Investigating the Effects of a Special Extract of Bacopa monnieri (CDRI 08) Hyperactivity and Inattention in Male Children and Adolescents: BACHI Study Protocol (ANZCTRN12612000827831). Nutrients, 7, 9931-9945. http://dx.doi.org/10.3390/nu7125507

[24] Stein, C., Hopfeld, J., Lau, H. and Klein, J. (2015) Effects of Ginkgo biloba Extract EGb 761, Donepezil and Their Combination on Central Cholinergic Function in Aged Rats. Journal of Pharmacy and Pharmaceutical Sciences, 18, 634-646. http://dx.doi.org/10.18433/J3WC8V

[25] SQUARE Herbal \& Nutraceuticals Ltd. Giloba. Accessed 10 January 2016. http://www.squarepharma.com.bd/downloads/Giloba.\%20DS.PDF

[26] Kongkeaw, C., Dilokthornsakul, P., Thanarangsarit, P., Limpeanchob, N. and Scholfield, C.N. (2014) Meta-Analysis of Randomized Controlled Trials on Cognitive Effects of Bacopa monnieri Extract. Journal of Ethnopharmacology, 151, 528-535. http://dx.doi.org/10.1016/j.jep.2013.11.008

[27] SQUARE Herbal \& Nutraceuticals Ltd. Monera. Accessed 10 January 2016. http://www.squarepharma.com.bd/downloads/Monera\%20DS.pdf

[28] Pandya, S.K. (2011) Understanding Brain, Mind and Soul: Contributions from Neurology and Neurosurgery. Mens Sana Monographs, 9, 129-149. http://dx.doi.org/10.4103/0973-1229.77431

[29] Kanwisher, N. (2010) Functional Specificity in the Human Brain: A Window into the Functional Architecture of the Mind. Proceedings of the National Academy of Sciences of the United States of America, 107, 11163-11170. 
http://dx.doi.org/10.1073/pnas.1005062107

[30] Grieve, S.M., Williams, L.M., Paul, R.H., Clark, C.R. and Gordon, E. (2007) Cognitive Aging, Executive Function, and Fractional Anisotropy: A Diffusion Tensor MR Imaging Study. American Journal of Neuroradiology, 28, 226-235.

[31] Kimberg, D.Y. and Farah, M.J. (1993) A Unified Account of Cognitive Impairments Following Frontal Lobe Damage: The Role of Working Memory in Complex, Organized Behavior. Journal of Experimental Psychology: General, 122, 411-428. http://dx.doi.org/10.1037/0096-3445.122.4.411

[32] Schacter, D.L., Gilbert, D.L. and Wegner, D.M. (2009) Psychology. Worth Publishers, New Work.

[33] Hergill, S.S., Bullmore, E.T., Brammer, M.J., Williams, S.C.R., Murray, R.M. and McGuire, P.K. (2001) A Functional MRI Study of Auditory Verbal Imagery. Psychological Medicine, 31, 241-253.

[34] Grill-Spector, K., Kourtzi, Z. and Kanwisher, N. (2001) The Lateral Occipital Complex and Its Role in Object Recognition. Vision Research, 41, 1409-1422. http://dx.doi.org/10.1016/S0042-6989(01)00073-6

[35] Wolf, U., Rapoport, M.J. and Schweizer, T.A. (2009) Evaluating the Affective Component of the Cerebellar Cognitive Affective Syndrome. The Journal of Neuropsychiatry and Clinical Neurosciences, 21, 245-253. http://dx.doi.org/10.1176/jnp.2009.21.3.245

[36] LaBar, K.S. and Cabeza, R. (2006) Cognitive Neuroscience of Emotional Memory. Nature Reviews Neuroscience, 7, 54-64. http://dx.doi.org/10.1038/nrn1825

[37] Oestreich, J. and Zakon, H.H. (2002) The Long-Term Resetting of a Brainstem Pacemaker Nucleus by Synaptic Input: A Model for Sensorimotor Adaptation. Journal of Neuroscience, 22, 8287-8296.

[38] Guyton, A.C. and Hall, J.E. (2006) Textbook of Medical Physiology. Elsevier Saunders, China.

[39] Strauss, E., Sherman, E.M.S. and Spreen, O. (2006) A Compendium of Neuropsychological Tests: Administration, Norms, and Commentary. Oxford University Press, Oxford.

[40] Thornhill, T. (2012) Art of the Matter: Mystery of Why Some People Are Good at Drawing and Others Aren’t Finally Revealed. http://www.dailymail.co.uk/sciencetech/article-2134898/Mystery-people-good-drawing-arent-revealed.html

[41] Quillin, K. and Thomas, S. (2015) Drawing-to-Learn: A Framework for Using Drawings to Promote Model-Based Reasoning in Biology. CBE—Life Sciences Education, 14, 1-16. http://dx.doi.org/10.1187/cbe.14-08-0128

[42] Brady, T.F., Konkle, T., Alvarez, G.A. and Oliva, A. (2008) Visual Long-Term Memory has a Massive Storage Capacity for Object Details. Proceedings of the National Academy of Sciences of the United States of America, 105, 1432514329. http://dx.doi.org/10.1073/pnas.0803390105

[43] Campbell, R., MacSweeney, M. and Waters, D. (2008) Sign Language and the Brain: A Review. Journal of Deaf Studies and Deaf Education, 13, 3-20. http://dx.doi.org/10.1093/deafed/enm035

[44] Zanto, T.P., Rubens, M.T., Thangavel, A. and Gazzaley, A. (2011) Causal Role of the Prefrontal Cortex in Top-Down Modulation of Visual Processing and Working Memory. Nature Neuroscience, 14, 656-661. http://dx.doi.org/10.1038/nn.2773

[45] Benton, A.L. (1968) Differential Behavioral Effects in Frontal Lobe Disease. Neuropsychologia, 6, 53-60. http://dx.doi.org/10.1016/0028-3932(68)90038-9

[46] Kipps, C.M. and Hodges, J.R. (2005) Cognitive Assessment for Clinicians. Journal of Neurology, Neurosurgery \& Psychiatry, 76, i22-i30. http://dx.doi.org/10.1136/jnnp.2004.059758

[47] Clouston, S.A.P., Kuh, D., Herd, P., Elliott, J., Richards, M. and Hofer, S.M. (2012) Benefits of Educational Attainment on Adult Fluid Cognition: International Evidence from Three Birth Cohorts. International Journal of Epidemiology, 41, 1729-1736. http://dx.doi.org/10.1093/ije/dys148

[48] Floyd, R.A. (1999) Antioxidants, Oxidative Stress, and Degenerative Neurological Disorders. Proceedings of the Society for Experimental Biology and Medicine, 222, 236-245. http://dx.doi.org/10.1046/j.1525-1373.1999.d01-140.x

[49] Price, C.J. (2000) The Anatomy of Language: Contributions from Functional Neuroimaging. Journal of Anatomy, 197, 335-359. http://dx.doi.org/10.1046/j.1469-7580.2000.19730335.x

[50] Stroop, J.R. (1935) Studies of Interference in Serial Verbal Reaction. Journal of Experimental Psychology, 18, 643-662. http://dx.doi.org/10.1037/h0054651

[51] McGivern, R.F., Adams, B., Handa, R.J. and Pineda, J.A. (2012) Men and Women Exhibit a Differential Bias for Processing Movement Versus Objects. PLoS ONE, 7, e32238. http://dx.doi.org/10.1371/journal.pone.0032238 


\section{Abbreviations}

MC: Matching capacity;

DI: Dissimilarity identification;

SM: Sense making;

GB: Ginkgo biloba;

BM: Bacopa monnieri;

PC: Placebo control;

PCC: Placebo control of the capsule;

PCS: Placebo control of the syrup;

AD: Alzheimer's disease;

PD: Parkinson's disease;

ALS: Amyotrophic lateral sclerosis;

DALYs: Disability-adjusted life-years;

SSC: Secondary school certificate;

HSC: Higher secondary certificate;

MT: Matching time;

MR: Memory retention;

TNCPs: Total number of correct portions matched by the subject;

TNPs: Total number of portions existing in the picture;

DFT: Dissimilarity finding time;

IA: Improved attention;

TNCDs: Total number of correct dissimilarities identified by the subject;

TNDs: Total number of dissimilarities presented in the picture;

SMT: Sense making time;

EC: Enhanced cognition;

TNCWs = Total number of correct words recognized by the subject;

TNWs = Total number of words offered in the paper;

IFJ: Inferior frontal junction.

\section{Submit or recommend next manuscript to SCIRP and we will provide best service for you:}

Accepting pre-submission inquiries through Email, Facebook, Linkedin, Twitter, etc A wide selection of journals (inclusive of 9 subjects, more than 200 journals)

Providing a 24-hour high-quality service

User-friendly online submission system

Fair and swift peer-review system

Efficient typesetting and proofreading procedure

Display of the result of downloads and visits, as well as the number of cited articles

Maximum dissemination of your research work

Submit your manuscript at: http://papersubmission.scirp.org/ 